\title{
Control of convection patterning and intensity in shallow cavities by harmonic vibrations
}

\author{
Marcello Lappa ${ }^{1}$ \\ ${ }^{1}$ Department of Mechanical and Aerospace Engineering, University of Strathclyde, James Weir Building, \\ 75 Montrose Street, Glasgow, G1 1XJ, UK - email: marcello.lappa@strath.ac.uk - marlappa@unina.it
}

\begin{abstract}
In this article the interplay among different types of flow (i.e. induced by driving forces of a different nature) is presented as a possible and "natural" means to control convection patterning and strength in shallow rectangular cavities of finite extent $(\mathrm{A}=$ length/height $=4)$ filled with a low Prandtl number liquid (silicon, Pr=0.01). A variety of results concerning the possible spatial structure of the "mixed" states of steady Buoyant, Marangoni and Vibrational convection are discussed with the express intent of supporting the optimization of future experiments to be performed onboard the International Space Station.
\end{abstract}

Keywords: Buoyant, Marangoni and Vibrational convection, Hybrid states, Flow control, Patterning Behaviour

\section{Introduction}

It is known that most technological processes for semiconductor crystal growth from the melt [horizontal and vertical Bridgman growth (see, e.g., Shemirani and Saghir, 2013), Czochralski method (e.g., Haslavsky et al., 2013), the floating-zone technique (Shevtsova et al., 2011a) and other processes (Lappa, 2004a, 2009 and 2012a; Oueslati et al., 2012; Sekhon et al., 2013)] require the application of thermal gradients in various directions; these gradients provide driving forces for free convection in all fluid phases involved (thermal gravitational flow in the presence of terrestrial gravity field and/or thermal surface-tension-driven fluid motion in the presence of a liquid-gas or liquid-liquid interface, Simanovskii and Kabov, 2012). The melt is therefore subject to varying heat- and mass-transfer conditions, which in recent years have been found to be directly or indirectly responsible for a vast majority of bulk deficiencies in single crystalline electronic materials.

Similar concepts can be applied to the case of macromolecular (protein) crystal growth (see, e.g., Carotenuto et al., 2002; Lappa et al., 2002; Lappa, 2003a). Crystals of such substances are typically grown from supersaturated solutions and a precipitant agent (a salt) and/or an imposed temperature difference are generally used to modulate the interaction between macromolecules, reducing their solubility. When salt concentration (or temperature) becomes sufficiently large (small) to make protein solubility smaller than the initial protein concentration, crystal nucleation and ensuing growth occur. These events, in turn, produce local depletion of protein, inducing gradients in its concentration distribution. As a natural consequence, undesired (lowering crystal quality) density-gradients driven convective instabilities may arise.

In the following, for simplicity, we will limit ourselves to considering the simplified situation (more relevant to the production of semiconductor crystals) in which free convection is produced solely by temperature differences (keeping in mind, however, that the mechanisms underlying the dynamics of macromolecular crystal growth are essentially the same, the role of temperature being replaced by that of solute concentration).

It is obvious that when a convective disturbance exists (i.e. a velocity field $\underline{\mathrm{V}}$ ), a distorted (with respect to a purely diffusive regime) thermo-fluid-dynamic (TFD) field is established; it is, in general, the difference between this field and an idealized purely conductive one $\left(\mathrm{TFD}_{\mathrm{diff}}\right.$ ) that gives a "measure" (at first order) of the effect that convection has on the final product $\mathrm{P}$ provided by the considered technological process ( $\mathrm{P}$ being, e.g., an inorganic or organic crystal or a solidified alloy). 
The TFD can be thought of as consisting of the aforementioned TFD $_{\text {diff }}$ plus an additional contribution due to convection. Having defined the "effect" of convection on the product as $\Delta \mathrm{P}$ and assumed the dependence $\mathrm{P}(\mathrm{TFD})$, one can always linearize and get $\Delta \mathrm{P}=\mathrm{P}-\mathrm{P}_{\text {diff }}=\mathrm{K}\left(\mathrm{TFD}-\mathrm{TFD}_{\text {diff }}\right)=\mathrm{K} \varepsilon$ ( $\mathrm{K}$ being a constant) and relate the overall product difference to the thermo-fluid-dynamic field distortions $\varepsilon=T F D-T^{2} D_{\text {diff }}$.

It is noteworthy how most of the time it is the ratio between the convective term over the diffusive one in the scalar transport equations that gives a measure of the TFD distortion $\varepsilon$ (TFD) (in the limit as $\underline{\mathrm{V}} \rightarrow \underline{0}$, $\mathrm{TFD} \rightarrow \mathrm{TFD}_{\text {diff }}$ and $\varepsilon(\mathrm{TFD}) \rightarrow 0$ ). The order of magnitude of this ratio is measured by the Peclet number $\left(\mathrm{Pe}=\mathrm{V} / \mathrm{V}_{\text {diff }}\right)$ where $\mathrm{V}_{\text {diff }}$ is the diffusive velocity $\left(\mathrm{V}_{\text {diff }}=\alpha / \mathrm{L}\right.$ in the case of thermal processes, where $\alpha$ is the thermal diffusivity and $\mathrm{L}$ a reference length) and $\mathrm{V}$ is a reference velocity characterizing the considered convective phenomena.

In conclusion, an estimation of the quality of the final product (via the related evaluation of the order of magnitude of the TFD distortion) can be done by an appropriate evaluation of the order of magnitude of the convective velocity, which, in turn, depends on the intensity of the surface-tension forces and/or on the acceleration level $\left(g_{0}=9.81\left[\mathrm{~m} / \mathrm{s}^{2}\right]\right.$ on the ground, steady and/or oscillatory accelerations prevailing on the considered microgravity platform in space).

In the case of thermal gravitational convection, the characteristic nondimensional number is the Rayleigh number $(\mathrm{Ra})$ that can be also regarded as a measure of the magnitude of the buoyancy velocity $\mathrm{V}_{\mathrm{g}}$ to the thermal diffusive velocity

$$
\begin{aligned}
& R a=\frac{g \beta_{T} \Delta T L^{3}}{v \alpha} \\
& \rightarrow \varepsilon=\varepsilon(\mathrm{Ra})
\end{aligned}
$$

where $v$ is the kinematic viscosity, $\alpha$ the aforementioned thermal diffusivity, $\beta_{\mathrm{T}}$ the thermal expansion coefficient, $\Delta \mathrm{T}$ a reference temperature gradient and, as anticipated, $\mathrm{L}$ a reference length for the geometrical configuration of interest (the associated Grashof number can be defined as $\mathrm{Ra} / \mathrm{Pr}$ where $\operatorname{Pr}$ is the well known Prandtl number $v / \alpha$ ).

In the case of thermal surface-tension-driven convection, the characteristic nondimensional number is the Marangoni number (Ma), defined as:

$$
\begin{aligned}
& M a=\frac{\sigma_{T} \Delta T L}{\mu \alpha} \\
& \rightarrow \varepsilon=\varepsilon(\mathrm{Ma})
\end{aligned}
$$

where $\sigma_{\mathrm{T}}$ is the surface tension derivative and $\mu$ the dynamic viscosity (the Reynolds number can be defined as $\mathrm{Ma} / \mathrm{Pr}$ ).

If the considered system is subjected to the action (usually referred to as "forcing") of harmonic vibrations $\underline{s}(t)=\operatorname{bsin}(\omega t) \underline{\hat{n}}$ along a generic direction $\underline{\hat{n}}$, it is known that a third type of fluid motion, generally referred to as "vibrational flow", can arise in the fluid. The related TFD distortion is, in general, a function of the nondimensional frequency $(\Omega)$ and displacement $(\Lambda)$ :

$$
\begin{aligned}
& \Omega=\frac{\omega L^{2}}{\alpha} \\
& \Lambda=b \frac{\beta_{T} \Delta T}{L} \\
& \rightarrow \varepsilon=\varepsilon(\Omega, \Lambda)
\end{aligned}
$$

where $b$ is the amplitude of the forced sinusoidal displacement and $\omega=2 \pi f$ ( $\mathrm{f}$ is the frequency). Such a periodic displacement is responsible for an acceleration:

$\underline{g}(t)=\underline{g}_{\omega} \sin (\omega \mathrm{t})$ 
where $\underline{g}_{\omega}=\mathrm{b} \omega^{2} \underline{\hat{n}}$.

(i.e. vibration of a system with frequency $\mathrm{f}$ and displacement amplitude $\mathrm{b}$ can be regarded as a sinusoidal gravity modulation with the same frequency and acceleration amplitude $b \omega^{2}$ and vice versa; accordingly, hereafter the terms "gravity modulation", "periodic acceleration", "vibration", "forcing” and "g-jitter" will be used as synonyms).

Eq. (4) represents the very idealized situation of a single frequency (also referred to as "monochromatic") disturbance; in practice, in natural environments (e.g., the International Space Station, ISS) real g-jitters are usually given by the superposition of disturbances with different amplitudes and frequencies (multifrequency or multicomponent spectrum), whose presence can be modeled by replacing eq. (4) with a series expansion. As an example, for the ISS, periodic, high frequency g-jitters, due to on-board machineries and natural frequencies excited by external forces, have amplitude and frequencies in the ranges $\left(10^{-6}<\mathrm{g}_{\omega} / \mathrm{g}_{0}<10^{-2}\right.$ and $0.1[\mathrm{~Hz}]<\mathrm{f}<300[\mathrm{~Hz}]$, respectively. Using the Boussinesq approximation, the related body force can be added to the momentum equation as an additional term, which in nondimensional form (having scaled time, velocity and temperature by $\mathrm{L}^{2} / \alpha, \alpha / \mathrm{L}$ and $\Delta \mathrm{T}$, respectively) and for the case of a monochromatic disturbance reads

$\operatorname{Pr} \frac{b \omega^{2} \beta_{T} \Delta T L^{3}}{v \alpha} T \sin \left(\frac{L^{2} \omega}{\alpha} t\right) \underline{\hat{n}}$

Hence, the nondimensional momentum equation can be written as:

$$
\frac{\partial \underline{V}}{a}=-\underline{\nabla} p-\underline{\nabla} \cdot[\underline{V} \underline{V}]+\operatorname{Pr} \nabla^{2} \underline{V}-\operatorname{Pr} R a T \underline{i}_{g}+\Lambda \Omega^{2} T \sin (\Omega t) \underline{\hat{n}}
$$

where $\underline{\underline{g}}_{\mathrm{g}}$ is the unit vector along the direction of gravity (obviously, the term -PrRaTi $\underline{\underline{i}}_{\mathrm{g}}$ is absent if the considered experiments are carried out on orbiting platforms where the steady gravity contribution is negligible).

The continuity and energy equations read:

$\underline{\nabla} \cdot \underline{V}=0$

and

$\frac{\partial T}{\partial t}=-\underline{\nabla} \cdot[V T]+\nabla^{2} T$

Obviously, such equations must be supplemented with the adequate kinematic and thermal boundary conditions, which in the general case read:

Kinematic conditions:

$\underline{\mathrm{V}}=0$ on solid wall(s)

$\frac{\partial \underline{V}_{S}}{\partial n}=-M a \underline{\nabla}_{S} T$ on a planar free liquid/gas interface

where $\mathrm{n}$ is the direction perpendicular to the free interface, $\underline{\nabla}_{S}$ the derivative tangential to the interface and $\underline{V}_{S}$ the surface velocity vector;

Thermal conditions:

$\mathrm{T}=\mathrm{T}_{\text {assigned }}$ on a conducting boundary 
$\frac{\partial T}{\partial n}=0$ for an adiabatic boundary

It must be also taken into account that in the limit of the socalled Gershuni's approximation (Gershuni and Lyubimov, 1998), i.e. under the assumptions of small amplitudes $(\Lambda<<1)$ and large frequencies of vibrations $(\Omega>>1)$, for a given Prandtl number, the steady vibrational convection depends only on one relevant dimensionless parameter, the vibrational Rayleigh number (often also simply referred to as the Gershuni number $G s)$ :

$G s=\frac{\left(b \omega \beta_{T} \Delta T L\right)^{2}}{2 v \alpha}=\frac{\left(\beta_{T} \Delta T L\right)^{2}}{2 v \alpha}\left(\frac{g}{\omega}\right)^{2}=\frac{\Omega^{2} \Lambda^{2}}{2 \operatorname{Pr}}$

$\rightarrow \varepsilon=\varepsilon(\mathrm{Gs})$

(that can be basically regarded as a combination of the nondimensional frequency and amplitude and of the Prandtl number). In such a case the equations given above can be rewritten as a closed set of equations for the time-averaged quantities: The time-averaged continuity, and energy equations remain unchanged; the time-averaged momentum equation can be cast in compact form as:

$$
\frac{\partial \overline{\underline{V}}}{\partial}=-\underline{\nabla} \bar{p}-\underline{\nabla} \cdot[\underline{\bar{V}} \bar{V}]+\operatorname{Pr} \nabla^{2} \underline{\bar{V}}-\operatorname{Pr} \operatorname{Ra} T \underline{i}_{g}+\operatorname{PrGs}[(\underline{w} \cdot \underline{\nabla} \bar{T}) \underline{\hat{n}}-\underline{w} \cdot \underline{\nabla} \underline{w}]
$$

Where the overbars are used to denote time-averaged quantities and $\underline{w}$ is an auxiliary potential function satisfying the equations:

$\underline{\nabla} \cdot \underline{w}=0$

$$
\underline{\nabla} \wedge \underline{w}=\underline{\nabla} \bar{T} \wedge \underline{\hat{n}} \rightarrow \nabla^{2} \underline{w}=-\underline{\nabla} \wedge(\underline{\nabla} \bar{T} \wedge \underline{\hat{n}})
$$

As an example, Fig. 1 shows the velocity field and related temperature distortion for a cavity with aspect ratio (horizontal length/depth) $\mathrm{A}=4$ and $\mathrm{Gs}=5 \times 10^{4}$.

In general, $\varepsilon=\varepsilon(\Omega, \Lambda)$ and in the context of the Gershuni's approximation, $\varepsilon=\varepsilon(\mathrm{Gs})$ with $\varepsilon(\mathrm{Gs}) \rightarrow 0$ for a given relative orientation of the applied temperature gradient and of the direction of the gravity modulation (the effect of high-frequency vibrations, like that of a steady acceleration, strongly depends on the direction of the vibration $\underline{\hat{n}}$ relative to the temperature gradient; in particular, vibrations parallel to the temperature gradient tend to maintain initial diffusive conditions).

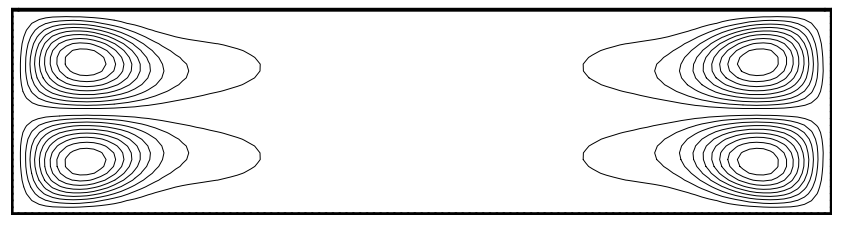

a)

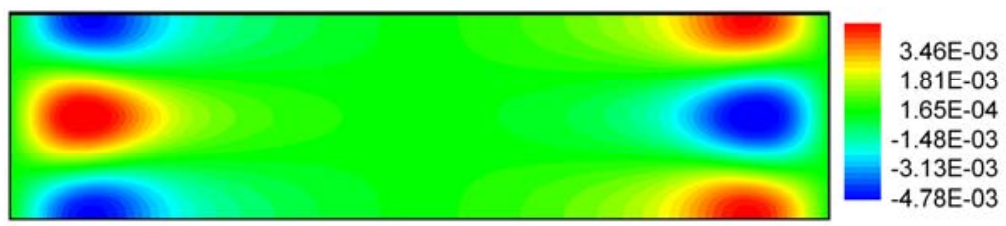

b)

Figs. 1: Thermovibrational convection for $\operatorname{Pr}=0.01$ (silicon) in a laterally heated enclosure with aspect ratio $A=4, G s=5 \times 10^{4}$, vibration perpendicular to the imposed temperature gradient (Gs based on the depth of the cavity, cold and hot sides on the left and on the right of each frame, respectively, upper and lower boundaries with adiabatic conditions): a) Velocity field $\left(\Psi_{\max }=0.20\right)$; b) $\mathrm{TFD}$ distortion $\delta \mathrm{T}=\left(\mathrm{T}-\mathrm{T}_{\mathrm{diff}}\right) / \Delta \mathrm{T}$, $\varepsilon_{\mathrm{T}}=\max (\delta \mathrm{T})=5.46 \times 10^{-3}$. 
It is known that the classical gravitational and Marangoni flows, governed by the Rayleigh and Marangoni number, respectively, can undergo undesired instabilities when the related characteristic number is increased (see, e.g., Lappa et al., 2003 and Lappa, 2003b and 2004b). These instabilities can strongly affect the flow and the transport of any solute or dispersed solid phase (Lappa, 2013a,b and 2014a and Kuhlmann et al., 2014). Thereby, they can strongly adversely affect the quality of the final product $P$ (such effects may be regarded as a "second order" contribution to be included into the dependence that relates $\mathrm{P}$ to the characteristic numbers via the TFD distortion, i.e. $\mathrm{P}=\mathrm{P}[\varepsilon(\mathrm{Ra}, \mathrm{Ma})]=\mathrm{P}(\mathrm{Ra}, \mathrm{Ma}))$.

There are several strategies that can be used to somehow "control" such instabilities (see, e.g, Gelfgat and Bar-Yoseph, 2001 for the use of magnetic fields and Shevtsova et al., 2014 for another variant based on the application of an externally-imposed shear).

Along the same lines, vibrational convection can be used to mitigate buoyancy and Marangoni convection (in general, there are regions of the parameter space where two different instability mechanisms can either reinforce or oppose each other). In particular, as pointed out by Birikh at al. (1993), the mean vibration force is a bulk driving action induced by temperature gradients normal to the vibration axis (i.e. thermovibrational convection arises when the isotherms are not perpendicular to the vibration axis), and the most remarkable feature of this force is that, if temperature distortions, with respect to the purely diffusive case, are induced by another type of convection, average vibrational flows arise in such a way as to permit the isotherms to turn and again become perpendicular to the vibration direction.

Examples of hardware available on the ISS that could be applied to suppress residual convection via harmonic lateral vibrations can be found in Trinchero et al. (2007) and Castagnolo et al. (2012).

The effects of vibrations on fluid motion for several possible initial and boundary conditions have been investigated theoretically by many authors (Kozlov, 1988; Monti and Savino, 1996; Savino and Monti, 1998; Kozlov and Selin, 2006; Shevtsova et al., 2011a,b; Parsa and Saghir, 2012; Ahadi and Saghir, 2012; Lyubimov et al., 2013; Lappa, 2014b; Lyubimova et al., 2014). Analytic solutions (valid in the limit as the considered system is infinitely extended along the direction of the imposed temperature gradient) for thermogravitational and Marangoni flows under the effect of vibrations have been discussed in a companion work (Lappa, 2012b). The present work considers similar cases for circumstances, however, of more effective practical and technological interest (two-dimensional finite-size enclosures), which require discretization and numerical solution of the governing eqs. (6-14).

\section{Mathematical model and numerical method}

The simulations discussed in the present work have been carried out within the framework of the Boussinesq approximation and the well-known SMAC numerical method for the case of incompressible fluids. The two-dimensional (2D) version of the code (the present numerical results are limited to the case of two-dimensional rectangular closed or open cavities) has been repeatedly validated through comparison with the results of the GAMM workshop (Roux, 1990). Second order accurate upwind and central difference schemes are used for the treatment of the convective and diffusive terms, respectively, in both momentum and energy equations. Thermal diffusive conditions and fluid at rest are assumed as initial conditions.

The aspect ratio (horizontal length/depth) considered is $\mathrm{A}=4$ for all the computations. This particular value of the aspect ratio is characteristic for some industrial processes and was used in the landmark experiments of Hurle et al. (1974), (the reader is also referred to the numerical studies by Winters (1988) and Skeldon et al. (1996).

According to a grid refinement study carried out for the specific value of the Prandtl number considered here, a mesh $120 \times 30$ has been used for all the simulations (percentage variations of computed quantities being less than $1.5 \%$ for a further $10 \%$ increase of the grid density). 
The article is organized as follows: some sections are devoted to illustrate (shortly) some "basic" features of classical convection mechanisms; other sections are specially focused on the interaction among different types of flow.

\section{Pure thermal gravitational flow}

\subsection{Lateral heating}

The simplest geometry among several crystal growth technologies has the horizontal configuration of the Bridgman method, where the convective flow is induced by the horizontal component of the temperature gradient (in an industrial setting, the crucible containing the molten crystal is withdrawn horizontally from a furnace, resulting in a horizontal temperature difference). This configuration has gained considerable academic interest because of its relative simplicity that allows progress to be made using analytical and numerical models of the flows (Batchelor, 1954; Cormack et al., 1974; Bontoux et al., 1986; Lappa, 2005 and 2007).

For $\mathrm{Pr}=0.01, \mathrm{~A}=4$ and a relatively low value of $\mathrm{Ra}=10^{3}$, the flow is simply given by a "twisted" elongated recirculation embracing three corotating (anticlockwise in the figures) vortices distributed along the horizontal extension of the enclosure (see Fig.2a, see also Gelfgat et al., 1999).

\subsection{Rayleigh-Bénard convection}

In Rayleigh-Bénard convection, when gravity is opposite to the density gradient $\underline{\nabla} \rho$, it has no effect (does not induce convective flows) for any value of its magnitude; when $\mathrm{g}$ is concurrent to $\underline{\nabla} \rho$ then TFD distortions arise only if the critical conditions for the onset of convection are exceeded.

For two-dimensional enclosures heated from below, in particular, it has been clearly illustrated by many investigators that there are several modes of the most dangerous perturbation that replace each other when the aspect ratio A is varied (Gelfgat, 1999). Within this context it should be mentioned that the increase of the aspect ratio A generally results in the decrease of the critical threshold (Luijkx and Platten, 1981) and in the increase of the number of two-dimensional rolls within the finite-size rectangular container.

In particular for $\mathrm{A}=4$, the number of rolls appearing at the primary instability of the motionless (quiescent) thermal diffusive state at $\mathrm{Ra}_{\mathrm{cr}} \cong 1810$, is $\mathrm{m}=4$ (see Fig. $4 \mathrm{a}$ ).

\section{Mixed buoyant/thermovibrational flows}

The case of combined buoyancy (induced by a steady gravity component) and thermovibrational convection is of great interest since it corresponds to the real situation occurring on the ISS.

This topic is made even more significant by the fact that, as explained before, time-periodic forcing has been also proposed as a possible means of dynamic control of flow instabilities on the ground. The character of natural buoyant convection in non-uniformly heated, rigidly contained inhomogeneous fluids, in fact, can be drastically altered by vibration of the container; accordingly, vibrational induced flow can potentially be used to influence and even control transport in some situations.

\subsection{Numerical results}

As a relevant example of all these behaviors Figures 2-5 show the interplay between vibrational (in the limit of the Gershuni's formulation, i.e. high frequency and small amplitude of imposed vibrations) and gravitational convection induced by steady gravity for different cases (system heated from the side or from below) in the canonical case of rectangular cavity with aspect ratio $\mathrm{A}=4$ filled with silicon melt $(\mathrm{Pr}=0.01)$ already considered as a paradigm model in Sect 3. The related discussion (given afterwards) proves that the interaction between these two types of convection, besides its effect on the magnitude of the resulting flow 
(enhanced or damped according to the relative direction of the vibrations and of the imposed temperature gradient) can also be used as a means to control "convection patterning", i.e. the spatial structure of the convection field (accordingly, values of the Rayleigh and vibrational Rayleigh number for which the "pure" states exhibit a comparable flow strength are selected for the examples since it is expected that in these cases, significant "competition" in determining the final convection pattern can occur between the different convective modes).

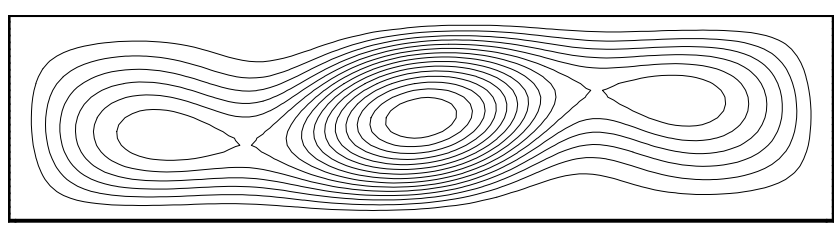

a)

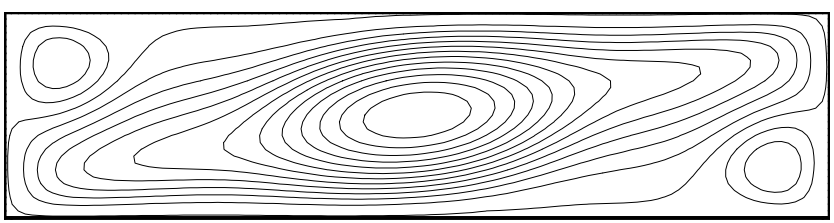

b)

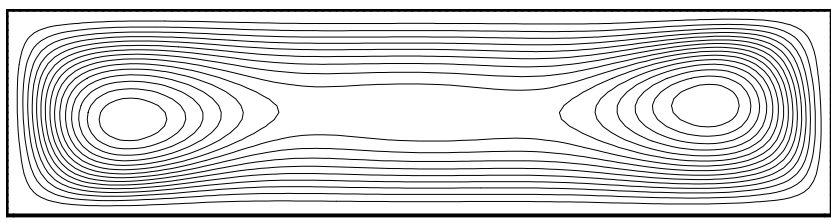

c)

Figs. 2: Thermal convection induced by coupled steady and oscillatory accelerations for $\operatorname{Pr}=0.01$ (silicon) in a laterally heated enclosure with aspect ratio $\mathrm{A}=4, \mathrm{Ra}=1 \times 10^{3}$ and $\mathrm{Gs}=5 \times 10^{4}$ ( $\mathrm{Ra}$ and $\mathrm{Gs}$ based on the depth of the cavity, cold and hot sides on the left and on the right of each frame, respectively, upper and lower boundaries with adiabatic conditions,): a) no vibrations imposed ( $\left.\Psi_{\max }=0.678\right)$; b) steady and oscillatory accelerations both acting in the vertical direction ( $\left.\Psi_{\max }=0.62\right)$; c) vibrations acting along the horizontal direction (stabilizing effect, $\Psi_{\max }=0.134$ )
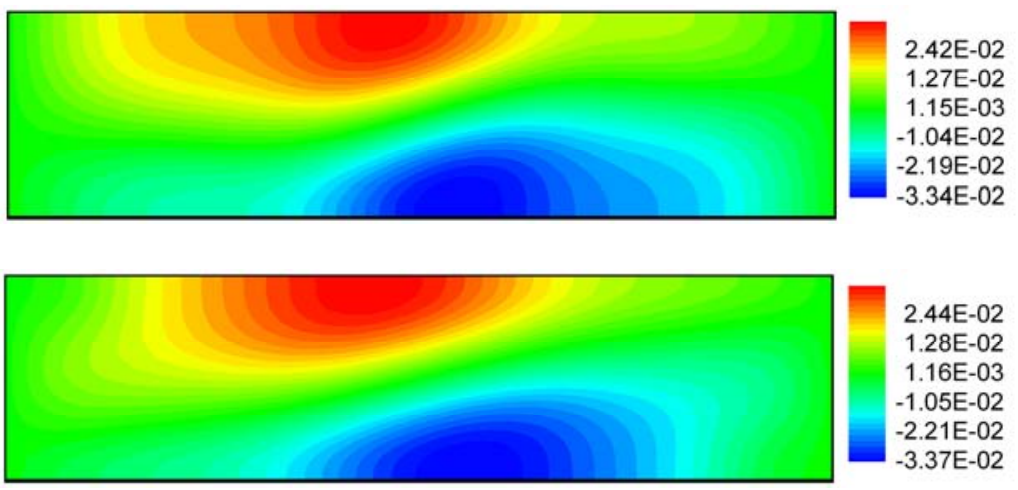

b)

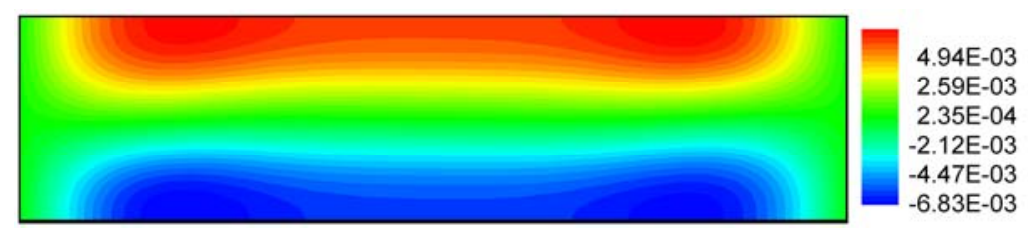

c)

Fig. 3: $\mathrm{TFD}$ distortion $\delta \mathrm{T}=\left(\mathrm{T}-\mathrm{T}_{\mathrm{diff}}\right) / \Delta \mathrm{T}\left(\varepsilon_{\mathrm{T}}=\max (\delta \mathrm{T})\right)$ for the same conditions corresponding to Fig. 2: a) $\varepsilon_{\mathrm{T}}=3.55 \times 10^{-2}$, b) $\varepsilon_{\mathrm{T}}=3.6 \times 10^{-2}$, c) $\varepsilon_{\mathrm{T}}=7.3 \times 10^{-3}$. 
When high frequency vibration is applied to the laterally heated enclosure along the direction perpendicular to its longest sides (hereafter simply referred to as the vertical direction), a main diagonal cell with two secondary vortices located in the upper left and in the lower right corners, respectively, appears (Fig. 2b). This structure is relatively similar to that obtained in the case of pure gravitational convection, the difference being given by the oblique diagonal orientation of the main roll and by the absence of minor corotating rolls embedded in the overall recirculation pattern with respect to the twisted structure of the elongated cell with three embedded minor rolls in frame (a) (in frame (b), in practice, two minor cells still exist but they are of a vibrational nature, counter-rotate with respect to the main one and, in addition, are confined to the corners).

In frame (c) (vibrations parallel to the temperature gradient) strong flow mitigation occurs (see the maximum value of the stream function given in the figure caption). Moreover, the twisted cell that characterizes the pure thermal gravitational flow in frame (a) is replaced by an elongated cell with straight streamlines almost parallel to the horizontal walls and only two corotating embedded anti-clockwise rolls near the left and right edges.

The mitigation and the opposite effect are clearly reflected in the TFD distortion distribution plotted in Figs. $3\left(\varepsilon_{\mathrm{T}}=3.55 \times 10^{-2}, 3.6 \times 10^{-2}\right.$ and $7.3 \times 10^{-3}$ in frames (a),(b) and (c), respectively).
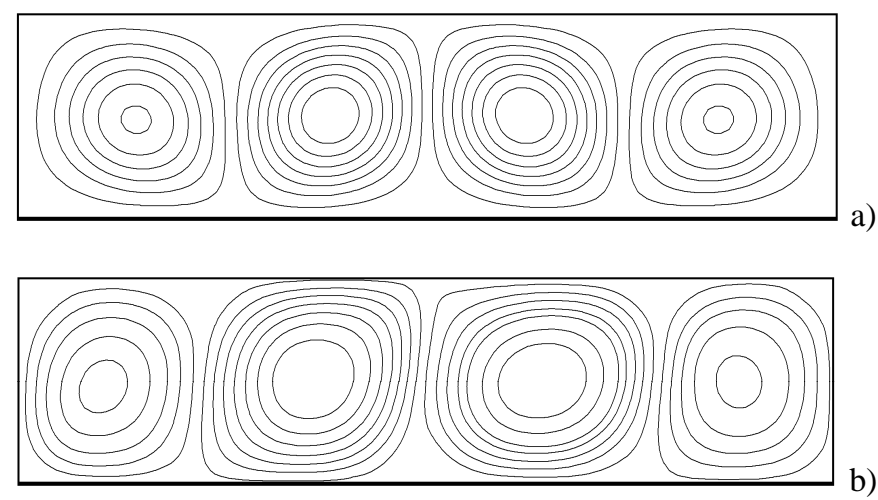

Figs. 4: Thermal convection and related temperature field induced by coupled steady and high-frequency oscillatory accelerations for $\mathrm{Pr}=0.01$ (silicon) in an enclosure heated from below (Rayleigh-Bénard configuration) with aspect ratio $\mathrm{A}=4, \mathrm{Ra}=2 \times 10^{3}$ and $\mathrm{Gs}=5 \times 10^{4}$ (Ra and Gs based on the depth of the cavity, vertical walls with adiabatic conditions); level $1 \rightarrow \mathrm{T}=0.0625$, level $15 \rightarrow \mathrm{T}=0.9375, \Delta_{\text {level }}=0.0625$ : a) no vibrations $\left(\Psi_{\max }=0.337\right)$; b) vibrations acting along the horizontal direction ( $\left.\Psi_{\max }=11.3\right)$.

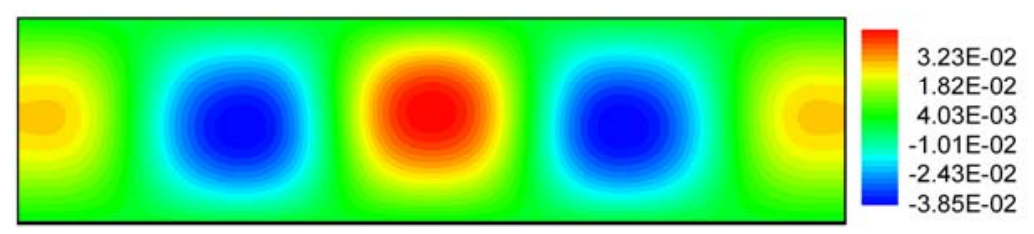

a)

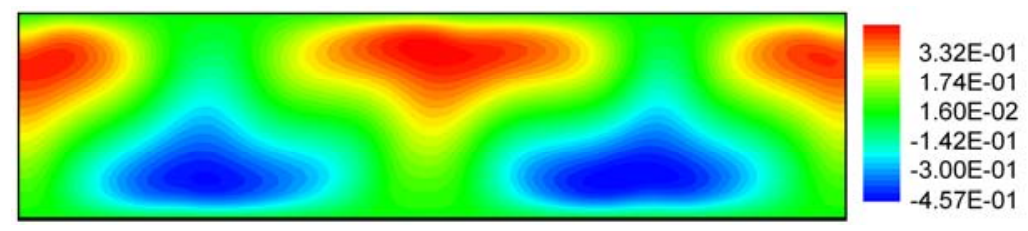

Fig. 5: $\mathrm{TFD}$ distortion $\delta \mathrm{T}=\left(\mathrm{T}-\mathrm{T}_{\mathrm{diff}}\right) / \Delta \mathrm{T}\left(\varepsilon_{\mathrm{T}}=\max (\delta \mathrm{T})\right)$ for the same conditions corresponding to Fig. 4 : a) $\varepsilon_{\mathrm{T}}=4.3 \times 10^{-2}$, b) $\varepsilon_{\mathrm{T}}=4.9 \times 10^{-1}$. 
For Rayleigh-Bénard convection (see Figs. 4 and 5), in an unexpected way, the application of acceleration in the horizontal direction (i.e. in direction perpendicular to the imposed temperature gradient), does not affect the qualitative structure of the velocity field (compare frames (a) and (b)), i.e. the emerging pattern. Thermovibrational flow simply strengthens the intensity of the basic Rayleigh-Bénard mode with four rolls (already discussed in Sect 3). This is reflected in both the streamfunction distribution and in the temperature field where the distortion with respect to the diffusive field becomes more significant (see Fig. $5, \varepsilon_{\mathrm{T}}=4.3 \times 10^{-2}$ and $4.9 \times 10^{-1}$ in frames (a) and (b), respectively)).

Most remarkably, when vibrations are imposed in the same direction of steady gravity (vertically), for Gs= $5 \times 10^{5}$, and $\mathrm{Ra}=2 \times 10^{3}$ it leads to complete suppression of convection (not shown) and consequently purely diffusive conditions are established (i.e. $\varepsilon_{\mathrm{T}}=0$ ).

\section{Pure thermal Marangoni flow}

It is well known that for small temperature differences $\Delta \mathrm{T}$, this flow in open cavities is steady and simply unicellular even for the case of elongated configurations. In the case of two-dimensional models, it appears as a unique large roll, whose axis is perpendicular to the temperature gradient and whose position changes according to the Prandtl number (at low Pr a recirculation roll develops near the cold wall, see, e.g., Fig. 6a, while at higher Pr the roll develops near the hot wall). It is also known (Ben Hadid and Roux, 1990), that for low values of the Reynolds or Marangoni number, this fluid configuration reaches a parallel flow state in the central region (core flow) of the cavity with the exception of an upwind region (close to the hot sidewall) in which the flow is accelerated and a downwind region (close to the cold sidewall) in which the flow is decelerated.

When the temperature gradient acting along the free surface reaches a critical value, various types of instabilities can be initiated in this system (see, e.g., Zhou and Huai, 2014 and references therein).

\section{Mixed Marangoni/thermovibrational convection}

Like buoyancy flow, also the thermal Marangoni flow can be strongly affected by imposed vibrations (see, e.g., Lappa, 2013c for the liquid-bridge problem).

Such a case is considered in this section. Besides the possible technological application, the interaction of thermal Marangoni flow and thermovibrational convection complements, from a theoretical point of view, the similar topics of mixed buoyancy/thermovibrational treated in Sect. 4.

In this case new mechanisms of flow generation arise, and by varying the vibrational parameters (i.e. amplitude or vibration frequency, or simply the vibrational Rayleigh number in the framework of the Gershuni's model), it is possible to change the flow characteristics (not only to damp the melt flow but also to generate a specific one).

\subsection{Numerical results}

As an example, Figs. 6-9 display in detail mixed Marangoni-thermovibrational and mixed BuoyantMarangoni-thermovibrational convective states for high-frequency imposed vibrations (Gershuni's approximation) and various conditions, respectively. 


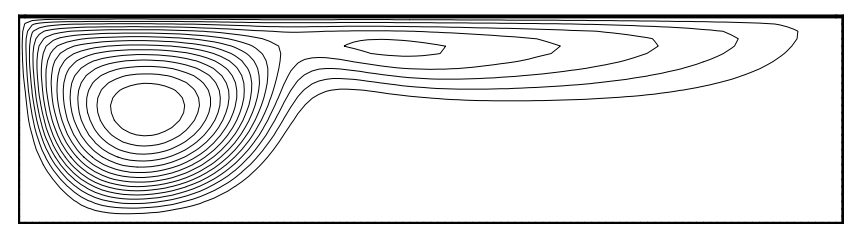

a)

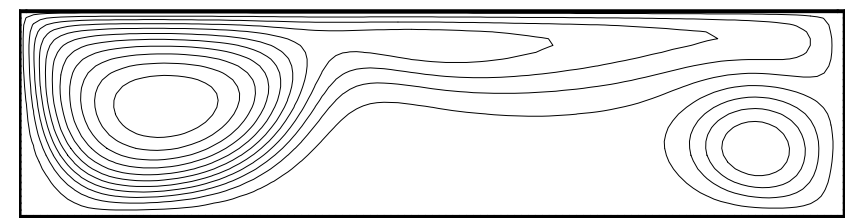

b)

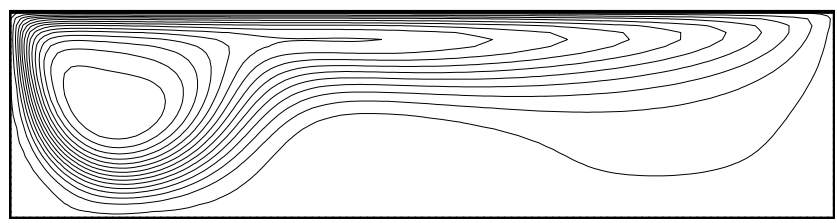

c)

Figs. 6: Mixed steady Marangoni-thermovibrational convection for $\operatorname{Pr}=0.01$ (silicon) in a laterally heated open cavity with aspect ratio $\mathrm{A}=4, \mathrm{Ma}=1 \times 10^{3}$ and $\mathrm{Gs}=5 \times 10^{4}$ (Ma based on the length and $\mathrm{Gs}$ based on the depth of the cavity, cold and hot sides on the left and on the right of each frame, respectively, upper and lower boundaries with adiabatic conditions,): a) no vibrations ( $\left.\Psi_{\max }=1.03\right)$; b) vibrations acting along the vertical direction $\left(\Psi_{\max }=1.01\right)$; c) vibrations acting along the horizontal direction (stabilizing effect, $\Psi_{\text {max }}=0.45$ )

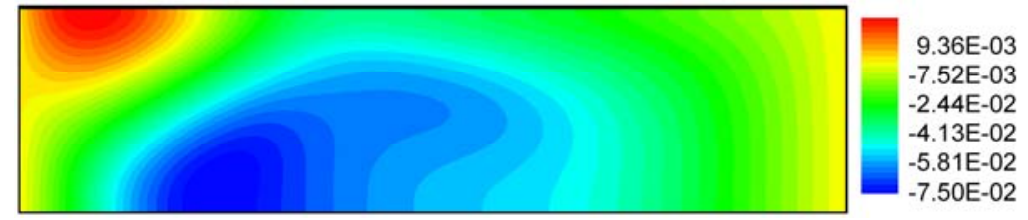

a)
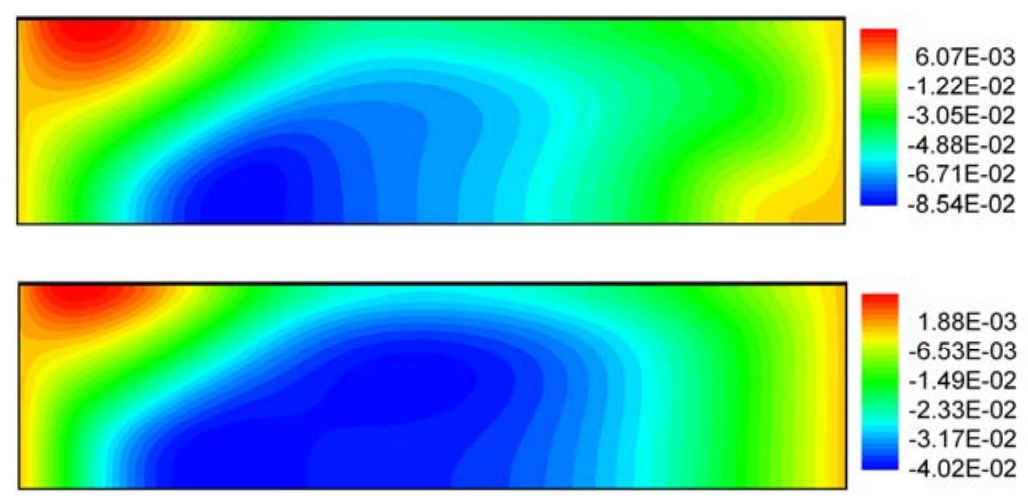

b)

Figs. 7: TFD distortion $\delta \mathrm{T}=\left(\mathrm{T}-\mathrm{T}_{\text {diff }}\right) / \Delta \mathrm{T}\left(\varepsilon_{\mathrm{T}}=\max (\delta \mathrm{T})\right)$ for the same conditions corresponding to Fig. 6: a) $\varepsilon_{\mathrm{T}}=7.8 \times 10^{-2}$, b) $\varepsilon_{\mathrm{T}}=8.9 \times 10^{-2}$, c) $\varepsilon_{\mathrm{T}}=4.1 \times 10^{-2}$. 


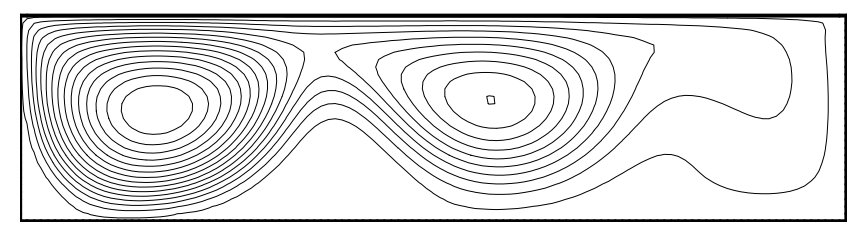

a)

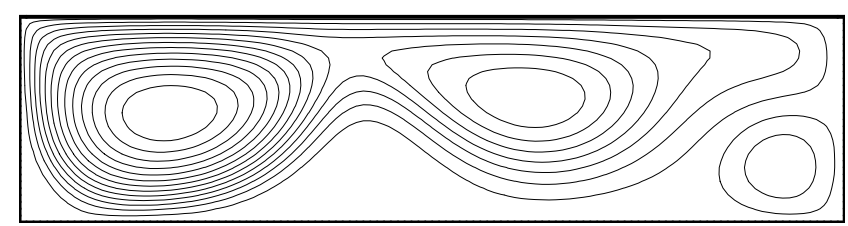

b)

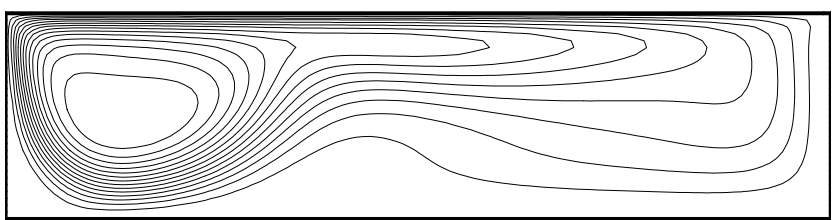

c)

Figs. 8: Structure of steady mixed Buoyant-Marangoni-Vibrational convection for $\mathrm{Pr}=0.01$ (silicon) and open cavity with aspect ratio $\mathrm{A}=4$ (cold and hot sides on the left and on the right of the figure, respectively, upper and lower boundaries with adiabatic conditions): $\mathrm{Ra}=1 \times 10^{3}$ and $\mathrm{Gs}=5 \times 10^{4}$ ( $\mathrm{Ra}$ and $\mathrm{Gs}$ based on the depth of the cavity) and $\mathrm{Ma}=1 \times 10^{3}$ (Ma based on the horizontal extension of the cavity): a) no vibrations $\left(\Psi_{\max }=1.64\right)$; b) vibrations acting in the vertical direction $\left(\Psi_{\max }=1.54\right)$; c) vibrations acting along the horizontal direction (stabilizing effect, $\Psi_{\max }=0.53$ )

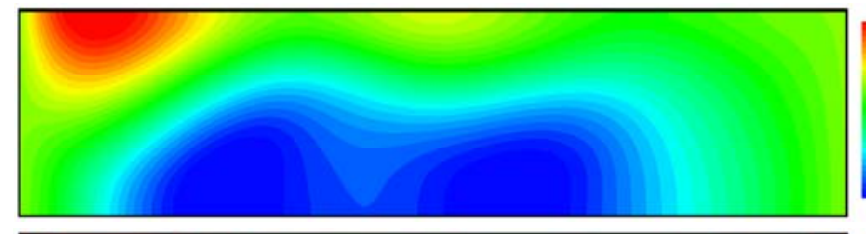

3.17E-02 8.06E-03

$-1.56 \mathrm{E}-02$

$-3.92 \mathrm{E}-02$

$-6.28 \mathrm{E}-02$

$-8.64 \mathrm{E}-02$ a)
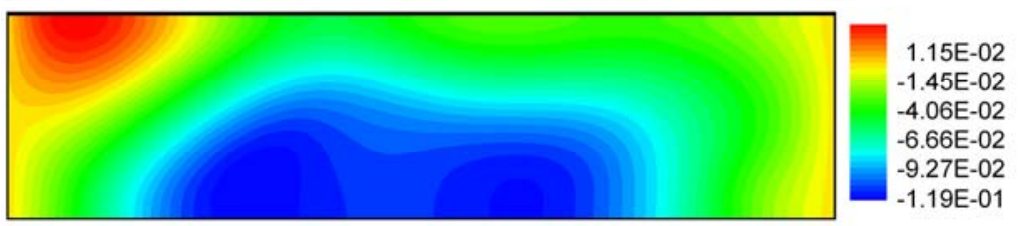

\section{a)}
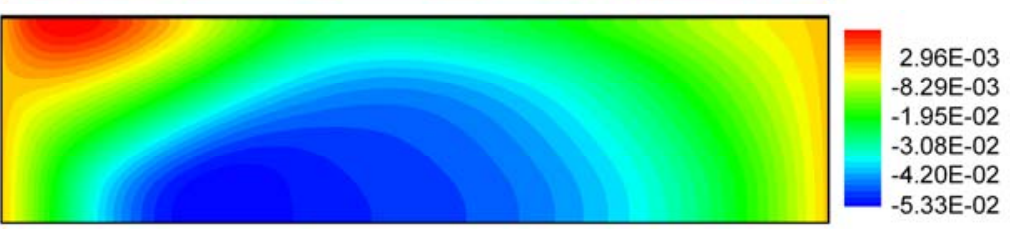

Figs. 9: TFD distortion $\delta \mathrm{T}=\left(\mathrm{T}-\mathrm{T}_{\mathrm{diff}}\right) / \Delta \mathrm{T}\left(\varepsilon_{\mathrm{T}}=\max (\delta \mathrm{T})\right)$ for the same conditions corresponding to Figs. 8: a) $\varepsilon_{\mathrm{T}}=9.6 \times 10^{-2}$, b) $\varepsilon_{\mathrm{T}}=1.24 \times 10^{-1}$, c) $\varepsilon_{\mathrm{T}}=5.5 \times 10^{-2}$.

Fig. 6a shows the pure Marangoni flow that occurs for $\mathrm{Ma}=1 \mathrm{x} 10^{3}$ with a strong anti-clockwise vortex near the cold wall (it is known that when the Marangoni number is increased above $1 \times 10^{3}$, the cell, that for lower values is extended throughout the horizontal length of the cavity, tends to be confined near the cold side). 
When vertical vibrations are applied to the cavity, the strength of the main Marangoni cell is slightly reduced (due to the non-linear interplay between the two types of convection) and a clockwise roll of a thermovibrational origin appears on the other side of the cavity (i.e. near the hot wall, see Fig. 6b). As a consequence of the thermovibrational effect, however the TFD distortions increase (see the value of $\varepsilon_{\mathrm{T}}$ in Figs. 7). With horizontal (mitigating) vibrations, the Marangoni flow is considerably weakened by the counteracting action of thermovibrational flow and the TFD distortion is reduced to about half of its value in the absence of forcing.

The case with all driving forces (buoyancy due to steady gravity, vibrational convection and Marangoni flow) acting at the same time is illustrated in Figs.8.

Without forcing a Marangoni cell is located near the cold wall and a corotating anti-clockwise circulation cell induced by buoyancy forces arises in the middle of the cavity. Both cells are anti-clockwise oriented and display a similar strength for the considered values of the Marangoni and Rayleigh numbers (Fig. 8a). Interestingly, in frame (b) (with vibrations in the vertical direction), three rolls of different nature are simultaneously present in the cavity: the aforementioned relatively strong Marangoni roll located in proximity to the cold wall, a central vortex due to classical thermal buoyancy and a lateral small cell of vibrational origin confined in the corner between the bottom adiabatic wall and the right hot side.

When forcing is considered in the horizontal direction, in a unexpected way, the action of vibrations is not limited to a simple damping of the intensity of the Marangoni flow; total suppression of the central cell of gravitational (steady) origin, in fact, occurs (in Figs. 8 and 9, compare frame (c) to frames (a) and (b)).

\section{Conclusions}

The use of imposed vibrations as a possible strategy to mitigate disturbances of different types as well as to "control" the related convection patterning in cavities of finite size has been addressed through "ad hoc" numerical simulations.

Apart from materials science and typical crystal-growth applications it is clear that the main ideas elaborated in the present work may be applied to a variety of other situations of technological interest (Lappa et al., 2004).

Beyond such practical applications, the present study complements from a purely theoretical point of view a companion analysis (Lappa, 2012b) in which mixed-convection states were treated in the framework of an analytical approach (under the limiting assumption of infinite plane-parallel flow, known to be a reasonable approximation for the core of real configurations, i.e. the region sufficiently away from the end regions to be considered non-influenced by sidewalls). We have seen here how finite-size effects (induced by solid walls perpendicular to the direction along which the system is more extended) have to be properly taken into account to get an effective prediction of the resulting patterns for both cases of pure and hybrid (with vibrations) convection.

\section{References}

Ahadi A.H. and Saghir M.Z., (2012), "Quasi Steady State Effect of Micro Vibration from Two Space Vehicles on Mixture During Thermodiffusion Experiment”, Fluid Dyn. Mater. Process., Vol. 8, No. 4, pp. 397-422.

Batchelor G. K., (1954), "Heat convection and buoyancy effects in fluids”,. Q. J. R. Met. Soc., Vol. 80, pp. 339-358.

Ben Hadid H., Roux B., (1990), “Thermocapillary convection in long horizontal layers of low-Prandtl number melts subject to a horizontal temperature gradient”, J. Fluid Mech., Vol. 221, pp. 77-103. 
Birikh R.V., Briskman V.A., Chernatynski V.I., Roux B., (1993), "Control of thermocapillary convection in a liquid bridge by high frequency vibrations”, Microgravity Q., Vol. 3, pp. 23-28.

Bontoux P., Roux B., Schiroky G. H., Markham B. L., Rosenberger F., (1986), “Convection in the vertical midplane of a horizontal cylinder. Comparison of two-dimensional approximations with threedimensional results”, Int. J. Heat Mass Transfer, Vol. 29, No. 2, pp. 227-240.

Carotenuto L., Piccolo C., Castagnolo D., Lappa M., Garcìa-Ruiz J.M., (2002), "Experimental observations and numerical modelling of diffusion-driven crystallisation processes”, Acta Crystallographica D, Vol., 58, pp. 1628-1632.

Castagnolo D., Lombardi S., Albanese C., Ceriello A., De Chiara G., Di Costanzo G., Lappa M., Piccolo C., Scognamiglio M., Sorrentino D., Tempesta S., Beestermoeller H.J., Cardano M., Lippi P., (2012), "Fluid Science Laboratory on board ISS: Two years of successful operations", 63rd International Astronautical Congress 2012 (Naples, Italy, 1-5 October 2012), paper number: IAC12.B6.1.5x15262, Volume 6, 2012, Pages 4949-4952 (ISBN: 978-162276979-7).

Cormack D. E., Leal L. G. and Imberger J., (1974), "Natural convection in a shallow cavity with differentially heated end walls, Part 1. Asymptotic Theory”, J. Fluid Mech., Vol. 65, pp. 209-229.

Gelfgat A. Yu. and Bar-Yoseph P. Z., (2001), "The effect of an external magnetic field on oscillatory instability of convective flows in a rectangular cavity”, Phys. Fluids, Vol. 13, No. 8, pp. 2269-2278.

Gelfgat A.Yu., (1999),”Different Modes of Rayleigh-Benard Instability in Two- and Three-Dimensional Rectangular Enclosures”, J. Comput. Phys., Vol. 156, pp. 300-324.

Gelfgat A.Yu., Bar-Yoseph P.Z. and Yarin A.L., (1999), "Stability of Multiple Steady States of Convection in Laterally Heated Cavities”, J. Fluid Mech., Vol. 388, pp. 315-334.

Gershuni G.Z. and Lyubimov D.V., (1998), “Thermal Vibrational Convection”, Wiley\&Sons (1998)

Haslavsky V., Miroshnichenko E., Kit E., and Gelfgat A. Yu., (2013), “Comparison and a Possible Source of Disagreement between Experimental and Numerical Results in a Czochralski Model”, Fluid Dyn. Mater. Process., Vol. 9, No.3, pp. 209-234.

Hurle D.T.J, Jakeman E., Johnson J.P., (1974), "Convective Temperature Oscillations in Molten Gallium”, J. Fluid Mech., Vol. 64, pp. 565-576.

Kozlov, V.G. (1988), "Vibrational thermal convection in a cavity executing high-frequency rocking motions”. Izv. AN SSSR. Mech. Zhidk. Gasa, Vol. 3, pp. 138-144. (Translated: Fluid Dyn., 23, 437442).

Kozlov, V.G. and Selin, N.V. (2006), "Pendulum thermal vibrational convection in a liquid layer with internal heat generation”, Fluid Dyn. Mater. Process., Vol. 2, No. 2, pp. 107-117.

Kuhlmann H.C., Lappa M., Melnikov D., Mukin R., Muldoon F.H., Pushkin D., Shevtsova V.S., and Ueno I., (2014), "The JEREMI-Project on thermocapillary convection in liquid bridges. Part A: Overview of Particle Accumulation Structures”, Fluid Dyn. Mater. Process., Vol. 10, No. 1, pp. 1-36.

Lappa M., (2003a), “Growth and Mutual Interference of Protein Seeds under reduced gravity conditions”, Physics of Fluids, Vol. 15, No. 4 , pp. 1046-1057.

Lappa M., (2003b), “Three-dimensional numerical simulation of Marangoni flow instabilities in floating zones laterally heated by an equatorial ring”, Physics of Fluids, Vol. 15, No. 3 , pp. 776-789.

Lappa M., (2004a), "Fluids, Materials and Microgravity: Numerical Techniques and Insights into the Physics”, 538 pages - ISBN-13: 978-0-08-044508-3, ISBN-10: 00-804-4508-X, Elsevier Science (2004, Oxford, England)

Lappa M., (2004b), "Combined effect of volume and gravity on the three-dimensional flow instability in non-cylindrical floating zones heated by an equatorial ring”, Physics of Fluids, Vol. 16, No. 2, pp. 331343. 
Lappa M., (2005), “On the nature and structure of possible three-dimensional steady flows in closed and open parallelepipedic and cubical containers under different heating conditions and driving forces”, Fluid Dyn. Mater. Process., Vol. 1, pp. 1-19.

Lappa M., (2007), "Secondary and oscillatory gravitational instabilities in canonical three-dimensional models of crystal growth from the melt, Part1: Rayleigh-Bènard systems”, Comptes Rendus Mécanique, Vol. 335, pp. 253-260. - M. Lappa, (2007), "Secondary and oscillatory gravitational instabilities in canonical three-dimensional models of crystal growth from the melt, Part2: Lateral heating and the Hadley circulation”, Comptes Rendus Mécanique, Vol. 335, pp. 261-268.

Lappa M., (2009). Thermal Convection: Patterns, Evolution and Stability (John Wiley \& Sons, Chichester, England, 2009).

Lappa M., (2012a), Rotating Thermal Flows in Natural and Industrial Processes (John Wiley \& Sons, Chichester, England, 2012).

Lappa M., (2012b), "Exact Solutions for Thermal Problems: Buoyancy, Marangoni, Vibrational and Magnetic-Field-Controlled Flows”, Review of Applied Physics, Vol. 1, No. 1, pp. 1-14.

Lappa M., (2013a), “On the Existence and Multiplicity of One-dimensional Solid Particle Attractors in Time-dependent Rayleigh-Bénard Convection”, Chaos, Vol. 23, No. 1, 013105 (9 pages).

Lappa M., (2013b), “Assessment of the role of axial vorticity in the formation of Particle Accumulation Structures (PAS) in supercritical Marangoni and hybrid thermocapillary-rotation-driven flows”, Physics of Fluids, Vol. 25, No. 1012101 (11 pages).

Lappa M., (2013c), "On the variety of particle accumulation structures under the effect of gjitters", Journal of Fluid Mechanics, Vol. 726, pp. 160-195.

Lappa M., (2014a), “Stationary Solid Particle Attractors in Standing Waves”, Physics of Fluids, Vol. 26, No. 1, 013305 (12 pages).

Lappa M., (2014b), “The patterning behaviour and accumulation of spherical particles in a vibrated nonisothermal liquid”, Physics of Fluids, Vol. 26

Lappa M., Castagnolo D., Carotenuto L., (2002), "Sensitivity of the non-linear dynamics of Lysozyme 'Liesegang Rings' to small asymmetries”, Physica A: Statistical Mechanics and its Applications, Vol. 314, No. 1-4, pp. $623-635$.

Lappa M., Piccolo C. Carotenuto L. (2004), "Mixed buoyant-Marangoni convection due to dissolution of a droplet in a liquid-liquid system with miscibility gap”, European Journal of Mechanics/B Fluids, Vol. 23, pp. 781-794.

Lappa M., Yasushiro S., Imaishi N., (2003), “3D numerical simulation of on ground Marangoni flow instabilities in liquid bridges of low Prandtl number fluid”, Int. J. Num. Meth. Heat Fluid Flow, Vol. 13, No. 3 , pp. 309-340.

Luijkx J. M., and Platten J. K., (1981), “On the onset of free convection in a rectangular channel”, $J$. Non-Equilibrium Thermodynam., Vol. 6, pp.141-148.

Lyubimov D. V., Popov D. M., Lyubimova T. P., (2013), “Stability of Plane-Parallel Pulsational Flow of Two Miscible Fluids under High Frequency Horizontal Vibrations”, Microgravity Science and Technology, Vol. 25, No. 4, pp. 231-236.

Lyubimova T., Beysens D., Gandikota G., Amiroudine S., (2014), "Vibration Effect on a Thermal Front Propagation in a Square Cavity Filled with Incompressible Fluid”, Microgravity Science and Technology, Vol. 26, No. 1, pp. 51-56.

Monti R. and Savino R., (1996), "Microgravity experiment acceleration tolerability on space orbiting laboratories”, Journal of spacecraft and rockets, Vol. 33, No. 5, pp. 707-716. 
Oueslati F.S., Bennacer R., Sammouda H., El Ganaoui, M., (2012), Analytical and numerical solutions for natural convection in a shallow cavity filled with two immiscible fluids: Shear stress action, Numerical Heat Transfer, Part A: Applications. Vol 62., No 8, pp. 605-623

Parsa A. and Saghir M.Z., (2012), "Fluid Flow Behavior of a Binary Mixture Under the Influence of External Disturbances Using Different Density Models”, Fluid Dyn. Mater. Process., Vol. 8, No. 1, pp. 27-50.

Roux, B., ed. (1990), "Numerical Simulation of oscillatory convection in low-Pr fluids”, a GAMM Workshop, Notes on numerical fluid mechanics, vol. 27. Vieweg.

Savino R. and Monti R., (1998), "Convection induced by residual-g and g-jitters in diffusion experiments”, Int. J. Heat Mass Transfer, Vol. 42, No. 1, pp. 111-126.

Sekhon M., Armour N. and Dost S, (2013), "Numerical Simulation of Liquid Phase Diffusion Growth of SiGe Single Crystals under Zero Gravity”, Fluid Dyn. Mater. Process., Vol. 9, No.4, pp. 331-352.

Shemirani M. M. and Saghir M. Z., (2013), “An Alternative Approach to Minimize the Convection in Growing a Large Diameter Single Bulk Crystal of Si0:25Ge0:75 Alloy in a Vertical Bridgman Furnace”, Fluid Dyn. Mater. Process., Vol. 9, No.1, pp. 11-22.

Shevtsova V., Gaponenko Y., Kuhlmann H.C., Lappa M., Lukasser M., Matsumoto S., Mialdun A., Montanero J.M., Nishino K., and Ueno I., (2014), “The JEREMI-Project on Thermocapillary Convection in Liquid Bridges. Part B: Impact of Co-axial Gas Flow”, Fluid Dyn. Mater. Process., Vol. 10, No. 2, pp. 197-240.

Shevtsova V., Mialdun A., Kawamura H., Ueno I., Nishino K., Lappa M., (2011a), "Onset of Hydrothermal Instability in Liquid Bridge. Experimental Benchmark”, Fluid Dyn. Mater. Process., Vol. 7, No. 1, pp. 1-28.

Shevtsova V., Mialdun A., Melnikov D., Ryzhkov I., Gaponenko Y., Saghir Z., Lyubimova T., and Legros J. C., (2011b), “The IVIDIL experiment onboard the ISS: Thermodiffusion in the presence of controlled vibrations,” Compt. Rend. Mécaniq., Vol. 339, No. (5), pp. 310-317.

Shevtsova V., Lyubimova T., Saghir Z., Melnikov D., Gaponenko Y., Sechenyh V., Legros J. C., and Mialdun A., (2011c), "IVIDIL: On-board g-jitters and diffusion controlled phenomena," J. Phys.: Conf. Ser. Vol. 327, 012031.

Simanovskii I. B. and Kabov O. A., (2012), "Nonlinear Convective Oscillations in Two-Layer Systems with Different Aspect Ratios”, Microgravity Science and Technology, Vol. 24, No. 2, pp. 127-137.

Skeldon A. C., Riley D. C. \& Cliffe K. A., (1996), “Convection in a low Prandtl number fluid”, J. Cryst. Growth, Vol. 162, pp. 95-106.

Trinchero G., Cardano M., Pensavalle E., Bassano E., Dell’Aversana P., Lappa M. and Tacconi M., (2007), "The Fluid Science Laboratory on the ISS Columbus module Performances and Operations", 3rd International Symposium on Physical Sciences in Space (ISPS 2007), Nara, Japan, 22- 26 October 2007

Winters K. H., (1988), “Oscillatory convection in liquid metals in a horizontal temperature gradient”, Int. J. Num. Meth. Eng., Vol. 25, pp. 401-414.

Zhou X. and Huai X., (2014), "Numerical Investigation of Thermocapillary Convection in a Liquid Layer with Free Surface”, Microgravity Science and Technology, Vol. 25, No. 6, pp. 335-341. 